\title{
Evaluación de los efectos mutagénicos/antimutagénicosde Luehea divaricata en ratones
}

Edith Alba Segovia, Romina Aidee Arrúa, Nathalia Celeste Barrozo, Rubén Darío Duré, Héctor David Nakayama, Inocencia Peralta

Laboratorio de Biotecnología. Centro Multidisciplinario de investigaciones y Tecnología-Dirección General de Investigación Científica y Tecnológica-Universidad Nacional de Asunción (CEMIT-DGICIT-UNA). Paraguay

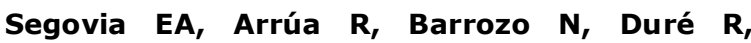 \\ Nakayama H, Peralta I. Evaluación de los efectos \\ mutagénicos/antimutagénicos de Luehea divaricata \\ en ratones. Mem. Inst. Investig. Cienc. Salud. \\ 2016;14(3):102-106
} Cómo referenciar este artículo/ How to reference this article:

\begin{abstract}
RE S U M E N
La Luehea divaricata Mart. conocida popularmente en el Paraguay como "ka'aovetî o ka'aovetîpytâ", es una planta nativa de la región utilizada como fitoterápico. El objetivo de este trabajo fue el de investigar el efecto protector del extracto acuoso de las hojas de $L$. divaricata frente al quimioterápico ciclofosfamida, en médula ósea de ratones. Los animales fueron divididos en 4 grupos de 5 animales cada uno; el Grupo I, control negativo, que recibió $200 \mu \mathrm{L}$ de agua, vía oral; el Grupo II, que recibió $200 \mu \mathrm{L}$ del extracto acuoso de L. divaricata; el Grupo III, que recibió $200 \mu \mathrm{L}$ del extracto acuoso de L. divaricata y ciclofosfamida, $50 \mathrm{mg} / \mathrm{Kg}$ de peso de animal y el Grupo IV, fue el control positivo, recibió ciclofosfamida $50 \mathrm{mg} / \mathrm{Kg} /$ peso de animal. El extracto acuoso de $L$. divaricata fue administrado por vía oral y el tratamiento se administró por $48 \mathrm{~h}$, la ciclofosfamida fue administrada por vía intraperitoneal $24 \mathrm{~h}$ antes del sacrifico del animal .Se extrajo la médula ósea de los animales y se realizó el ensayo de micronúcleo en todas las muestras. Los resultados indicaron que el extracto acuoso de L. divaricata no presentó actividad mutagénica, cuando combinado con el agente mutagénico induce una reducción de hasta $49 \%$ en la frecuencia de micronúcleos, al ser comparado con el tratamiento con ciclofosfamida, sugiriendo un potencial efecto protector frente a agentes tóxicos.
\end{abstract}

Palabras clave: Luehea divaricata, micronúcleo, efecto protector.

\section{Evaluation of the mutagenic / antimutagenic effects of Luehea divaricata in mice}

\section{A B S T R A C T}

The Luehea divaricata Mart. is a plant native, popularly known in Paraguay as "ovetîka'a" or "ovetîka'aPytá" of the region used as herbal medicines. The aim of this study was to investigate the protective effect of aqueous extract from the leaves of L. divaricata versus cyclophosphamide chemotherapy. The animals were divided in 4 groups and each group have 5 animals, Group I, negative control, which received 200 uL of water, orally; Group II, which received $200 \mathrm{uL}$ of aqueous extract of L. divaricata; Group III, which received $200 \mathrm{uL}$ of the aqueous extract of L. divaricata and cyclophosphamide, $50 \mathrm{mg} / \mathrm{kg}$ animal weight; Group IV, positive control, cyclophosphamide received $50 \mathrm{mg} / \mathrm{kg} /$ animal weight. The aqueous extract of L. divaricata was orally administered and the treatment was administered for 48 hs, cyclophosphamide was administered intraperitoneal and had 24 hours of treatment. Bone marrow of the animals is removed and micronucleus assay was performed on all samples. The results indicated that aqueous extract of $L$. divaricata showed no mutagenic activity and when combined with the mutagen agent induces a reduction of up to $41 \%$ in the frequency of micronucleus, when compared to treatment with cyclophosphamide, suggesting a potential protective effect against toxic agents.

Key words: Luehea divaricata, micronucleus, protector effect 


\section{INTRODUCCIÓN}

Las plantas medicinales son utilizadas en muchos países del mundo. En el Paraguay el consumo de plantas medicinales y aromáticas es tradicional y generalizado, una costumbre que se heredó de los tiempos de los guaraníes $(1,2)$. Entre estas plantas tenemos la Luehea divaricata Mart., conocida popularmente en el Paraguay como ka'aovetî o ka'aovetîpytâ; a nivel regional tiene una distribución que abarca Brasil, Uruguay y Argentina (3).La flor se utiliza como anticoqueluche, antitusígeno, antiasmático, antidiabético, carmitivo, contra el ácido úrico (4).A pesar de su amplia utilización en la medicina popular, son pocos los estudios sobre los posibles efectos secundarios de los extractos de esta especie. Entre los trabajos realizados encontramos un estudio del extracto acuoso de las hojas de $L$. divaricata en Salmonella typhimurium, en el que se observó que tiene un efecto mutagénico en la cepa TA 98, al ser activada con la fracción microsomal S9 (5). Estudios del potencial mutagénico en Drosophila melanogaster han mostrado que el extracto acuoso de la corteza de L. divaricata fue negativo en los cruzamientos Standard y de alta bioactivación; al combinarse el extracto acuoso de la corteza con un mutágeno conocido, la Doxorubicina (DRX), se ha observado un efecto reductor de la acción mutagénica de manera significativa, al reducir significativamente las frecuencias de manchas de pelos mutantes en las alas de $D$. melanogaster, al ser comparadas con los datos obtenidos en el tratamiento de DRX solo (6), lo que indicaría un efecto protector del extracto de L.divaricata frente a agentes mutagénicos.En estudios previos, realizados por nuestro equipo de trabajo, hemos encontrado que el extracto acuoso de $L$. divaricata no presentó efectos genotóxicos en células de médula ósea de ratones, cuando fueron tratadas con el extracto acuoso por 48 hs (7). Los extractos de plantas medicinales pueden presentar efectos tóxicos en las células de los organismos tratados (8) o presentar efectos protectores frente a daños hepatotóxicos, inflamatorios (9) o genotóxicos, lo que indica que tienen el efecto de inducir la disminución de daño en el ADN o aumentar el mecanismo de reparación en células o tejidos expuestos a agentes físicos, químicos o biológicos $(10,11,12,13)$. Considerando que extractos de plantas medicinales o comestibles son utilizadas como protectores de daños a tejidos del organismo se podría utilizar el extracto de L. divaricata con esta finalidad. El objetivo de este trabajo fue el de analizar el efecto protector del extracto acuoso de la L. divaricata en células de médula ósea de ratones, utilizando el Test de Micronúcleo (14).

\section{MATERIALES Y MÉTODOS}

Especie vegetal. Las muestras de L. divaricata fueron colectadas en la ciudad de San Lorenzo e identificadas por la Lic. Biol. Bonifacia de Bertoni, del Herbario de la Facultad de Ciencias Exactas y Naturales (FACEN) de la Universidad Nacional de Asunción (UNA). EI número de colecta de la autora principal es 02 y el "voucher" de la especie fue depositado en el Herbario de FACEN-UNA.

Preparación del extracto de la planta. Para obtener el extracto acuoso de las hojas de $L$. divaricata, se colocaron $10 \mathrm{gr}$ de hojas maceradas, previamente deshidratadas a $40^{\circ} \mathrm{C}$ en estufa, en $100 \mathrm{~mL}$ de agua destilada, a $80^{\circ} \mathrm{C}$, que se mantuvo por 10 minutos sobre la plancha calefactora, se dejó enfriar y se filtró con dos sistemas de filtros, filtro común y filtro de $0,45 \mu \mathrm{m}$. El trabajo de investigación fue realizado en el laboratorio de Biotecnología (CEMIT-DGICT-UNA).

Animales. Para este estudio se utilizaron 20 (veinte) ratones Swiss albinos sanos, hembras, de 20 a $30 \mathrm{gr}$ de peso y entre 6 a 12 semanas de vida. Los animales fueron mantenidos en el laboratorio de Biotecnología por una semana para su aclimatación con comida y agua ad libitum. Teniendo en cuenta los códigos de ética para trabajos de experimentación con animales de laboratorio, se ha evitado todo sufrimiento innecesario del animal y se ha empleado el mínimo número requerido con valor estadísticamente aceptable.

Técnica para análisis in vivo de micronúcleos

Los animales fueron divididos en 4 grupos de 5 animales; Grupo1:control negativo, al cual se administró $200 \mu \mathrm{L}$ de agua potable, vía oral (v.o.); Grupo 2:control positivo, al cual se administró $50 \mathrm{mg} / \mathrm{Kg}$ peso de animal vía intraperitoneal (i.p.)deciclofosfamida (CAS No:6055-19-2, Sigma); ambos tratamientos fueron 24 hs antes del sacrificio. Grupo 3:al cual se administró el extracto acuoso de la planta $L$ divaricata, $200 \mu \mathrm{L}$, i.p., 48 y 24 hs antes del sacrificio, Grupo 4: al cual se administró $200 \mathrm{uL}$ del extracto acuoso v.o. a48 y 
$24 \mathrm{~h}$ antes del sacrifico y $50 \mathrm{mg} / \mathrm{Kg}$ peso del animal, $24 \mathrm{~h}$ antes del sacrificio. Se sacrificaron los animales, se retiraron los fémures y se removió la médula ósea con suero fetal bovino (FBS); mantenido a $37^{\circ} \mathrm{C}$. Se homogenizaron las muestras y se transfirierona tubos cónicos, se centrifugaron a $1000 \mathrm{rpm}$ durante 5 minutos. Se desechó el sobrenadante dejando un pequeño volumen para resuspender las células. Se resuspendieron las células restantes, se colocaron 1-2 gotas sobre una lámina de cristal limpia y seca y se realizó el extendido, se dejaron secar las muestras y se fijaron las mismas en metanol absoluto. Se tiñeron las muestras con Giemsa al 4\%. Se analizaron las muestras al microscopio óptico con aumento de inmersión y se contaron 1000 Eritrocitos Policromáticos (EPCs) incluyendo los que presentaron Micronúcleos (EPCMNs). Para el análisis de citotoxicidad se contaron 200 eritrocitos por animal tratado para establecer el porcentaje de EPCs; la disminución de este porcentaje en los animales tratados es indicativa de toxicidad o supresión de células de médula ósea. Se utilizaron 5 animales por volumen de extracto analizado, así como en los controles del ensayo. Los análisis estadísticos se realizaron con la ayuda del software Excel 2007 ( Microsoft Excel), Past 3 (15) y BioEstat 5.3 (16). La reducción de daño se calculó de las medias del Índice de daño (ID) en los grupos tratados con CP, extracto y CP (Ld) y grupo Control negativo (CTL-), conforme a la fórmula(17): \%Reducción=Media ID Ld-Media CP/Media Ld-Media CTL-.

\section{RESULTADOS}

En la tabla 1 se muestra las frecuencias (números) de Micronúcleos (MNs) en células de médula ósea de ratones tratados con el extracto acuoso de L. divaricata, sola o en combinación con el quimioterápico ciclofosfamida. Fueron analizadas 5.000 células por grupode tratamiento, la frecuencia de MNs en el grupo de L. divaricata $200 \mu \mathrm{L}$ fue de $0,58 \%$ sobre el total de células analizadas y no presentó diferencia estadísticamente significativa cuando es comparada con la frecuencia encontrada en el control negativo (0.44). La frecuencia de MNs en el Grupo 3 presentó una frecuencia de MNs de 1,54\%, y del Grupo 4 fue de $2,6 \%$, en 5.000 células analizadas en cada grupo;ambas frecuencias fueron estadísticamente significativa cuando fueron comparadas con la frecuencia del Grupo 1. Se encontraron diferencias significativas al compararse las frecuencias del Grupo 3 y el Grupo 4 ; donde hemos verificado un \% de Reducción en la frecuencia deMNs de $49 \%$ en el Grupo3 y que fue estadísticamente significativo.

Tabla 1. Frecuencia de eritrocitos policromáticos micronucleados, Media y Desvío Standard $(\mathrm{SD})$, en médula ósea de ratones.

\begin{tabular}{|c|c|c|c|c|c|c|c|c|}
\hline \multirow[t]{2}{*}{ Tratamiento } & \multicolumn{5}{|c|}{ Frecuencia de MN por animal } & \multirow{2}{*}{ EPC } & \multirow{2}{*}{$\begin{array}{l}\text { Número de EPCMN } \\
(\%)\end{array}$} & \multirow{2}{*}{$\begin{array}{l}\text { EPC } \\
\text { Media } \pm \text { SD }\end{array}$} \\
\hline & $\mathbf{A}_{1}$ & $\mathbf{A}_{2}$ & $\mathbf{A}_{3}$ & $\mathbf{A}_{4}$ & $\mathbf{A}_{5}$ & & & \\
\hline Grupo 1 & 6 & 5 & 8 & 1 & 2 & 5000 & $22(0,44)^{a}$ & $4,4 \pm 2,88$ \\
\hline Grupo 2 & 3 & 5 & 9 & 9 & 3 & 5000 & $29(0,58)^{a}$ & $5,8 \pm 3,03$ \\
\hline Grupo 3 & 13 & 14 & 21 & 16 & 13 & 5000 & $77(1,54)^{\mathrm{a}^{*} \mathrm{~b}^{*}}$ & $15,4 \pm 3,36^{*}$ \\
\hline Grupo 4 & 24 & 27 & 15 & 38 & 26 & 5000 & $130(2,6)^{\mathrm{a}^{*}}$ & $26 \pm 8,22 *$ \\
\hline
\end{tabular}

comparado con el Grupo 4. *Diferencia estadísticamente significativa( $p \leq 0.05)$

\section{DISCUSIÓN}

Este estudio investigó la actividad protectora de la planta L. divaricatafrente a un agente genotóxico conocido y el ensayo de Micronúcleo, utilizado como biomarcador, provee información sobre el potencial genotóxico de los agentes que son testados. Este ensayo permite la detección del efecto clastogénico (quiebras de cromosomas) o aneugénico (pérdida de cromosomas enteros). Este efecto se verifica analizando las modificaciones en la frecuencia de micronúcleos, es altamente sensible, fácil de realizar, de bajo costo (18) y se utiliza tanto para verificar los efectos tóxicos o los efectos protectores de agentes químicos, físicos o biológicos Estos ensayos son válidos tanto para realizar un estudio de los efectos genotóxicos (clastogénicos o aneugénicos) de algunas substancias como para estudiar los posibles efectos protectores de otras $(19,20)$. La Ciclofosfamida (CP), es un Mem. Inst. Investig. Cienc. Salud. 2016;14(3):102-106 
quimioterápico que produce aberraciones cromosómicasin vitro (21) e in vivo y micronúcleos en las células de la médula ósea de ratones tratados con una dosis simple del fármaco $(22,23)$.

La acción del extracto de $L$. divaricata ya fue estudiada en células tumorales, con acción citotóxica sobre las mismas y con acción anti fúngica en Staphylococcus aureus. Felicio et al. (6) han demostrado los efectos protectores del extracto frente al daño producido por un mutágeno en células de $D$. melanogaster. En este trabajo los animales pre-tratados con el extracto acuoso de L. divaricata y luego tratados con CP han mostrado una disminución significativa en la frecuencia de MNs en células de médula ósea, lo que se debería al efecto protector del extracto, por disminución de daño sobre el DNA y/o por activación de los mecanismos de reparación de las células. Los extractos de plantas medicinales o comestibles se utilizan como terapéuticos o protectores frente a daños producidos en el organismo por agentes exógenos, su eficacia debe ser demostrada en estudios científicos; el extracto acuoso de L. divaricata analizado en este trabajo ha mostrado tener un efecto reductor del daño al genoma inducido por un mutágeno conocido; para sugerir su utilización como protector se recomienda otros estudios complementares, tanto in vivo como in vitro.

\section{REFERENCIAS BIBLIOGRÁFICAS}

1. Paraguay: Informe Nacional para la Conferencia Técnica Internacional de la FAO sobre los Recursos Fitogenéticos (Leipzing, 1996), Asunción, junio 1995.

2. González -Torres D. 2005. Catálogo de plantas medicinales (y alimenticias y útiles) usadas en Paraguay. 2005. Editorial Servilibro, AsunciónParaguay.

3. Molfino JF. Nota sobre las especies argentinas de Género Luehea.Com. Museo nacional de historia natural de Buenos Aires, 1923 2(5):53-60 in: Di Sapio O.A. y Gattuso M.A. Análisis micrográfico para la identificación de la corteza de Luehea divaricata Mart. ("sota caballo, Arbol de San Francisco") (Tiliaceae). 1996. ROJASIANA 3 (2):177-185.

4. Pin A, González G, Marín G, Céspedes G, Cretton S, Christen P, Rouguet D. Plantas medicinales del jardín Botánico de Asunción. 2009. Asociación Etnobotánica paraguaya 441.

5. Vargas VM, Guidobono RR, Henriques JA. Genotoxicity of plantextracts. Mem Inst Oswaldo Cruz. 1991;86Suppl 2:67-70.

6. Felicio LP, Silva EM, Ribeiro $V$, Miranda CT, Vieira IL, Passos DC, Ferreira AK, ValeCR, Lima DC, Carvalho S, Nunes WB. Mutagenic potential and modulatory effects of the medicinal plant Luehea divaricata (Malvaceae) in somatic cells of Drosophila melanogaster: SMART/wing.Genetics and Molecular Research. 2011 Jan 4;10(1):16-24.

7. Duré RD, Nakayama HD, Ibarra $P$, Segovia E. Estudios del potencial genotóxico, in vivo, de Luehea divaricata (ka'áovetî o ka 'áovetîpytâ). Jornadas de Jóvenes Investigadores- Presentación oral.

Julio 2012.Universidad Nacional de Asunción.

8. Maistro EL, Mota SF, Lima EB, Bernardes BM, Goulart FC. Genotoxicity and mutagenicity of Rosmarinus officinalis (Labiatae) essential oil in mammalian cells in vivo. 2010. Genetics and Molecular Research 9 (4): 2113-22.

9. Kandimalla et al. Effect of $Z$. jujube on Liver Toxicity and Inflammation, Frontiers in Pharmacology 11September 2016doi: 10.3389/fphar.2016.00298 Volume7 | Article 298

10. Ghoneum MK, Badr El-Din N, Abdel Fattah SM, Tolentino L. Arabinoxylan rice bran (MGN-3/Biobran) provides protection against whole-body $\mathrm{Y}$ irradiation in mice via restoration of hematopoietic tissues. Journal of Radiation Research, 2013, 54, 419429.

11. Madrigal-Santillán E, Fragoso-Antonio S, Valadez-Vega, C, Solano-Solano, G, Zúñiga Pérez $C$, SánchezGutiérrez, M, Izquierdo-Vega, J. A, Gutiérrez-Salinas, J, Esquivel-Soto, J, Esquivel-Chirino, C, Teresa SumayaMartínez, T, Fregoso-Aguilar, T, Mendoza-Pérez, J and MoralesGonzález, J.A. Investigation on the Protective Effects of Cranberry Against the DNA Damage Induced by Benzo [a] pyrene. Molecules 2012, 17, 4435-51.

12. Fan ZL, Wang ZY, Zuo LL, Tian SQ. Protective effect of anthocyanins from lingonberry on radiation-induced damages Int J Environ Res Public Health. 2012 Dec. 18;9(12):4732-43. doi: $10.3390 /$ ijerph9124732.

13. Melo-Cavalcante AA, Dantas SM, Leite Ade S, Matos LA, e Sousa JM, PicadaJN, da Silva J. In vivo antigenotoxic and anticlastogenic 
effects of fresh and processed cashew (Anacardiumoccidentale) apple juices. Med Food. 2011 Jul-Aug;14(7-8):7928. Epub 2011 May 25.

14. Schmid W. The micronuclei test. Mutat Res. 1975; 31:9-15.

15. Hammer $\varnothing$, Harper DAT, Ryan PD. PAST: Paleontological statistics software package for education and data analysis. Palaeontologia Electronica. $\quad 2001 ; \quad 4(1)$ : 9pp. http://palaeoelectronica.org/2001_1/past/issue1_0 1.htm.

16. Ayres, M, Ayres Júnior M, Ayres DL. \& Santos AA. 2007. BIOESTAT Aplicações estatísticas nas áreas das ciênciasbio-médicas. Ong Mamiraua. Belém, PA.

17. Devi HP, Mazumder PB. Methanolic Extract of Curcuma caesia Roxb. Prevents the Toxicity Caused by Cyclophosphamide to Bone Marrow Cells, Liver and Kidney of Mice. Pharmacognosy Research, 2016, JanMarch, 8(1):43-9.

18. Patlolla AK, Hussain SM, Schlager RJ J, Patlolla S, Tchounwou PB. Comparative Study of the Clastogenicity of Functionalized and Non-functionalized Multi-walled Carbon Nanotubes in Bone Marrow Cells of Swiss-Webster Mice. Environ Toxicol. 2010 25(6): 608-21.

19. Premkumar K, Bowlus CL. 2003. Ascorbic Acid Reduces The Frequency Of Iron Induced Micronuclei In Bone Marrow Cells of Mice. Mutat Res. 2003; Dec 9;542(1-2):99-103.
20. de Carvalho NC, FrydbergCorrêaAngeloni MJ, Dimer Leffa D, Moreira J, Nicolau V, de AguiarAmaral $P$, Rossatto $A E$, Moraes de Andrade $V$. Evaluation of the genotoxic and antigenotoxic potential of Melissa officinalis in mice. Genet Mol Biol. 2011 Apr-Jun; 34(2): 290-7.

21. De Salvia R, Fiore $M$, Aglitti T, Festa F, Ricordy R, Cozzi R. Inhibitory action of melatonin on $\mathrm{H} 2 \mathrm{O} 2-$ and cyclophosphamide-induced DNA damage.. Mutagenesis. 1999; 14 (1) pp.107-12.

22. Franke SI, Prá D, Erdtmann B, Henriques JA, da Silva J. Influence of orange juice over the genotoxicity induced by alkylating agents: an in vivo analysis. Mutagenesis. $2005 \mathrm{Jul}$; 20(4):279-83.

23. Rehman MU, Tahir M, Ali F, Qamar W, Lateef A, Khan R, Quaiyoom A, OdayO-Hamiza, Sultana S. Cyclophosphamide-induced nephrotoxicity, genotoxicity, and damage in kidney genomic DNA of Swiss albino mice: the protective effect of Ellagic acid. Mol Cell Biochem. 2012 Jun;365(1-2):119-27. 